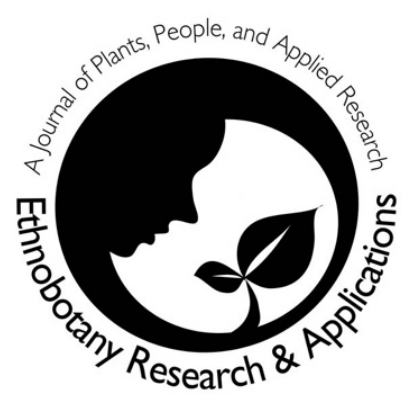

Ethnobotanique et

caractérisation variétale

de Jatropha curcas L.

dans les savanes

soudaniennes du Nord-

Cameroun

Ethnobotany and varietal

characterization of

Jatropha curcas L. in the

Sudanese savannas of

North-Cameroon

Anguessin Benjamine and Mapongmetsem Pierre Marie

\section{Notes on Ethnobotany}

\section{Résumé}

Contexte: Le réchauffement climatique grandissant et le coût élevé des hydrocarbures ont motivé le regain d'intérêt pour la recherche de nouvelles sources d'énergie non polluantes. L'espèce Jatropha curcas qui présente des importances énergétiques est peu connue. L'objet du présent travail vise à identifier les différents usages ethnobotaniques ainsi que les variétés de Jatropha curcas afin de valoriser l'espèce dans la région.

Méthodes: Les enquêtes ethnobotaniques ont été réalisées auprès de 200 ménages qui manipulent quotidiennement l'espèce Jatropha curcas. Les individus de Jatropha curcas après dénombrement, ont fait l'objet d'analyse dendrométrique en composantes principales (ACP) et en classification ascendante hiérarchisée $(\mathrm{CAH})$. Les paramètres relevés sont : le diamètre à hauteur de poitrine, la hauteur de l'arbuste, la surface foliaire et la longueur du pétiole. Le programme mis à profit est le logiciel (B).

\section{Correspondence \\ Anguessin Benjamine ${ }^{1 *}$, Mapongmetsem Pierre Marie $^{2}$ \\ ${ }^{1}$ Anguessin Benjamine, Département des Sciences Environnementales, Ecole Nationale Supérieure Polytechnique, Université de Maroua, BP 46, Cameroun. \\ ${ }^{2}$ Mapongmetsem Pierre Marie, Laboratoire de Biodiversité et Développement Durable, Faculté des Sciences, Université de Ngaoundéré, BP 454, Cameroun. \\ *Corresponding Author: kpdeben@yahoo.fr \\ Ethnobotany Research \& Applications 21:04 (2021)}

Résultats: les résultats révèlent que Jatropha curcas est utilisé en médecine traditionnelle et soigne plusieurs pathologies: gonococcie, jaunisse, brulures, parasitoses intestinales.... Tapinantus sp. qui parasite l'espèce est très apprécié par les 
populations pour ses propriétés médico-magiques malgré son impact négatif sur la plante. Jatropha curcas est également intégré dans les systèmes agroforestiers et joue d'importants rôles dans la délimitation des champs, les haies vives, les clôtures végétales, la base foncière et la lutte contre l'érosion des sols. Selon la longueur du pétiole, la surface foliaire, la hauteur de l'arbuste et le diamètre à hauteur de poitrine, on distingue quatre variétés de Jatropha curcas dans la région. Des analyses moléculaires pourront permettre d'approfondir l'analyse taxonomique dans la perspective de promouvoir la variété la plus performante.

Conclusion: La culture de Jatropha curcas pourrait générer des revenus aux producteurs et permettrait de faire face aux enjeux énergétiques du pays.

Mots clés:: ethnobotanique, dendrométrie, biocarburant, Jatropha curcas, variété, NordCameroun

\section{Abstract}

Background: Growing global warming and the high cost of hydrocarbons have increased the interest in the search for new non-polluting energy sources. Little is known about the species Jatropha curcas which is rich of energy potential. The purpose of this work is to identify the different ethnobotanical uses as well as the varieties of Jatropha curcas in order to promote the species in the region.

Methods : Ethnobotanical surveys were carried out among 200 households that handle the species Jatropha curcas on a daily basis. The individuals of Jatropha curcas after enumeration were subjected to dendrometric analysis in principal components (PCA) and in ascending hierarchical classification $(\mathrm{CAH})$. The parameters recorded were: diameter at breast height, shrub height, leaf area and petiole length. The program used was logicel $®$.

Results : the results show that Jatropha curcas is used in traditional medicine and treats several pathologies: gonococcal disease, jaundice, burns, intestinal parasitosis, etc. Tapinantus sp. which parasitizes the species is highly appreciated by populations for its medico-magical properties despite its negative impact on the plant. Jatropha curcas is also integrated into agroforestry systems and plays important roles in field delineation, hedgerows, plant fencing, land base and soil erosion control. Depending on the length of the petiole, the leaf area, the height of the shrub and the diameter at breast height, there are four varieties of Jatropha curcas in the region. Molecular analyzes will allow the taxonomic analysis to be deepened with a view to promoting the best performing variety.
Conclusions: The Jatropha curcas culture could generate income for producers and make it possible to face the country's energy challenges.

Keywords: ethnobotany, dendrometry, biofuel, Jatropha curcas, variety, Northern Cameroon

\section{Contexte}

La mesure du danger que constitue l'émission des gaz à effet de serre par les énergies fossiles en particulier les produits pétroliers a amené les pouvoirs publics, les institutions nationales et internationales et les organismes non gouvernementaux à repenser leur politique en matière d'énergie. Ces gaz sont à l'origine de graves perturbations environnementales conduisant aux changements climatiques observés de plus en plus et qui hypothèquent l'équilibre écologique dans les différentes régions du monde (Berchmans \& Hirata 2008, Gbosségnon 2007). De même le coût de plus en plus élevé des hydrocarbures et la perspective d'un épuisement de cette ressource énergétique non renouvelable a amené les Etats du monde à réfléchir au développement de nouvelles sources d'énergie écologiquement propre et économiquement rentable (Ballerini 2006). Parmi ces sources d'énergie renouvelables, les bioénergies en général et les biocarburants en particulier sont celles qui attirent l'attention des responsables politiques locaux et internationaux compte tenu du fait que la filière carburant est celle qui consomme le plus de produits pétroliers et qui produit le plus de gaz nuisibles à l'environnement et à la santé (Gbosségnon 2007). Par ailleurs le développement des biocarburants a créé de nouvelles filières agricoles et pourraient offrir de nouveaux créneaux porteurs pour les agriculteurs des pays en voie de développement: bois, fibres, produits alimentaires végétaux et animaux pour l'alimentation et la pharmacopée (Mapongmetsem et al. 1997, Noumi \& Anguessin 2010, Sokpon \& Lejoly 1996).

La présence d'arbres dans les espaces cultivés constitue une caractéristique fondamentale des paysages agraires dans la région du NordCameroun (Kossoumna 2008). Ce système de gestion des terres agricoles est une pratique très ancienne en milieux tropicaux (Torquebiau 2007). Pour Molenaar et Kessler (2008), l'agroforesterie procure de nombreux avantages tels que la protection des cultures, des animaux d'élevage, des sols et des cours d'eau, la diversification des revenus agricoles, la conservation de la biodiversité, la séquestration du carbone et l'embellissement du paysage. C'est ainsi que l'espèce Jatropha curcas à haut potentiel de production de biocarburant mais mal connue au Cameroun est souvent intégrée dans les systèmes de production paysannes. Sa culture 
pourrait générer des revenus aux producteurs et permettrait de faire face aux enjeux énergétiques du pays. Le manque d'informations sur le potentiel disponible notamment les différentes variétés et l'usage de Jatropha curcas est un handicape important pour la promotion de ce matériel biologique. L'objet du présent travail vise à identifier les différents usages ethnobotaniques ainsi que les variétés de Jatropha curcas afin de valoriser l'espèce dans la région.

\section{Materiels et Methodes \\ Choix et description du site}

La zone d'étude se situe dans les savanes soudanosahéliennes du Nord-Cameroun. Les sites explorés sont ceux de Guider, Sorawel, Djabi, Badjengo et leurs environs (Fig. 1). La région du Nord-Cameroun est située entre $7^{\circ}$ et $10^{\circ}$ de latitude Nord et entre $12^{\circ}$ et $16^{\circ}$ de longitude Est (Kossoumna 2008). Ces sites font partis de la vaste vallée de la Bénoué qui s'étend sur environ $250 \mathrm{~km}$. Le climat de la région : est du type soudano-sahélien intermédiaire entre le climat sahélo-soudanien du Diamaré et le climat soudano-guinéen de l'Adamaoua (Humbel 1971).

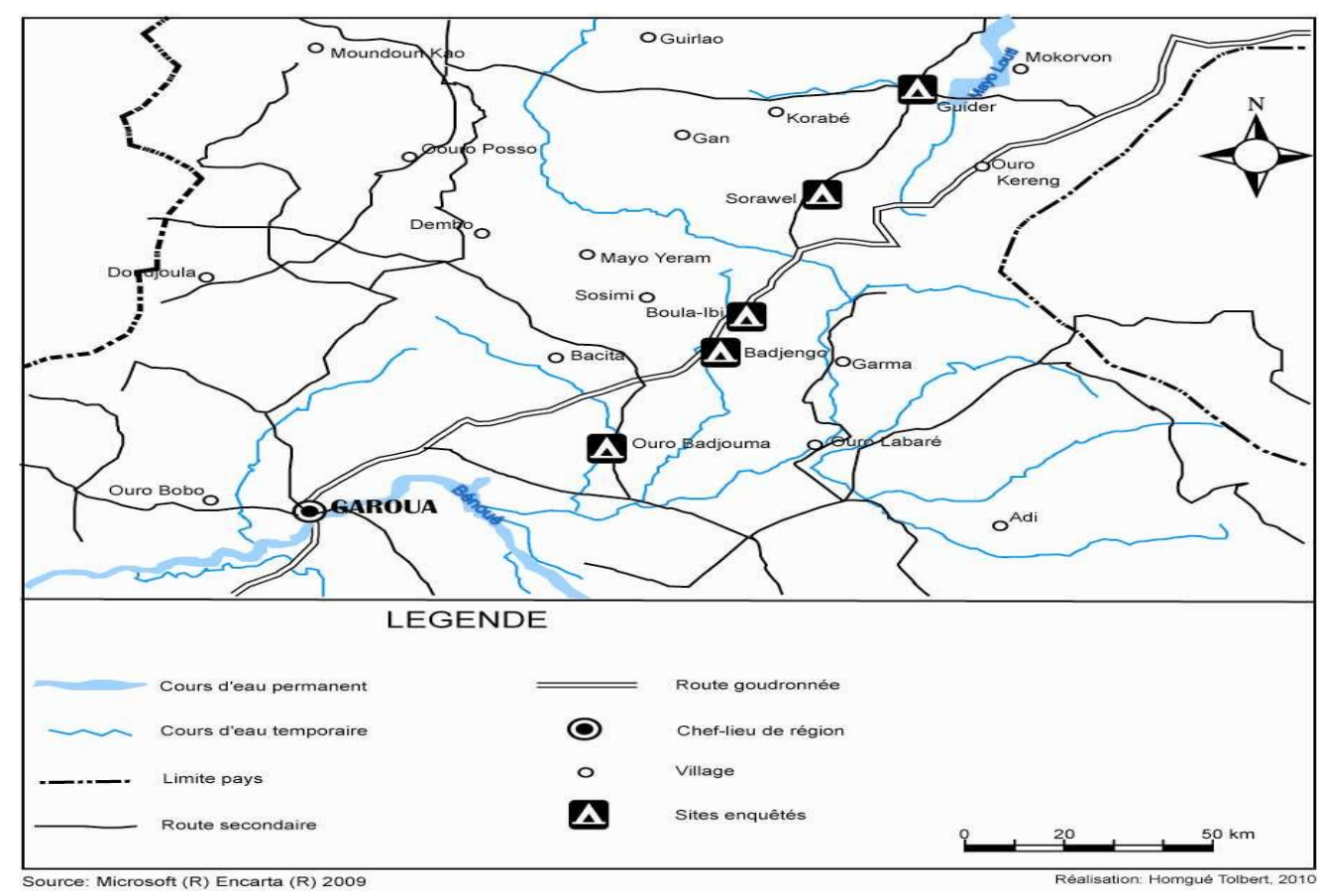

Figure 1. Carte de localisation des sites d'étude

Figure 1. Location map of study $s$

\section{Méthodologie}

Les enquêtes ethnobotaniques se sont déroulées chez les groupes ethniques suivants : Guidar, Daba, Peulhs et Guiziga. Ce sont les groupes les plus représentatifs des sites explorés (Guider, Sorawel, Djabi, Badjengo). Le choix des ménages est guidé par la présence d'au moins un pied de Jatropha curcas dans la concession. L'essentiel des investigations sur le terrain s'est fait par interview au moyen d'un questionnaire administré face à face et comprenant des questions ouvertes qui ont permis aux paysans de répondre délibérément ; des questions fermées auxquelles les informateurs répondaient par oui ou par non et des questions orientées auxquelles les répondants portaient leur choix sur une ou deux des réponses proposées. Les enquêtes ont été menées auprès de 200 ménages soit 50 personnes par ethnie. Tous les individus de Jatropha curcas ont été comptés dans chaque ménage. De même les systèmes d'utilisation des terres dans lesquelles cette Euphorbiaceae intervenait ont été identifiés et décrits. En plus chaque individu de J. curcas a fait l'objet d'une analyse dendrométrique. Les paramètres relevés sont : le diamètre à hauteur de poitrine, la hauteur de l'arbuste, la surface foliaire et la longueur du pétiole. Pour chacun de ces paramètres 50 individus ont été exploités par site. Les données collectées ont fait l'objet d'analyse en composantes principales (ACP) et d'une classification ascendante hiérarchisée $(\mathrm{CAH})$. Le programme mis à profit est le logiciel $\circledR$.

\section{Résultats}

Ethnobotanique de Jatropha curcas L.

Noms vernaculaires et origine de Jatropha curcas La population de la région du Nord-Cameroun exploite le Jatropha depuis de décennies, preuve de l'existence du nom vernaculaire dans le lexique 
dialectal des différentes ethnies de la région. Chaque ethnie donne au genre Jatropha un nom. Ainsi il est connu sous plusieurs noms : dondoli (en Guidar), colcoladjé (en fulfuldé) chez les Peuhls, paycoladjé (en Daba) et dimbick chez les Guiziga. La distinction entre les espèces porte sur la couleur des organes végétatifs (la couleur verte pour l'espèce Jatropha curcas et la couleur rouge pour l'espèce Jatropha gossypifolia). Tous ces noms vernaculaires n'ont pas une explication particulière. Malgré l'ancienneté de $J$. curcas dans la région, l'origine de la plante reste très peu connue. La provenance varie d'un groupe ethnique à l'autre (Tab. 1) ; 44,5\% de la population interrogée déclare l'avoir obtenu chez les voisins tandis que $33 \%$ ignore sa provenance. Quelques-uns l'ont cependant hérité de leurs parents. C'est le cas des Daba (30\%) et des Guidar $(12 \%)$.

Tableau 1. Origine de Jatropha curcas

Table 1. Origin of Jatropha curcas

\begin{tabular}{|c|c|c|c|c|}
\hline & \multicolumn{4}{|c|}{ Origine de Jatropha curcas (\%) } \\
\hline Ethnies & Héritage & Sauvage & Voisins & Ignorance \\
\hline Guidar & 12 & 24 & 42 & 22 \\
\hline Daba & 30 & 16 & 40 & 14 \\
\hline Peuhls & 0 & 0 & 46 & 54 \\
\hline Guiziga & 0 & 08 & 50 & 42 \\
\hline Moyenne & 10,5 & 12 & 44,5 & 33 \\
\hline
\end{tabular}

Potentialités de Jatropha curcas L. dans la région Les différents usages de la plante dans la région varient avec les groupes ethniques. La plante est utilisée comme médicament, dans la protection des cultures et dans la délimitation des terrains et champs. D'une manière générale, il apparaît que la principale valorisation de la plante repose sur la médecine traditionnelle.

Usage médicinal de Jatropha curcas $L$.

Presque toutes les parties de la plante se prêtent à l'utilisation médicale (feuilles, tige, racines, fruits et gui). Les modes de préparation des différents médicaments sont : la décoction, l'infusion, la macération, l'extrait de sève et l'onction (Tab. 2).

La sève de la plante est utilisée comme antiseptique, elle permet une guérison rapide après application sur les blessures. Les feuilles écrasées ou bouillies puis collées sur les blessures et les brûlures assurent la guérison rapide de ces dernières. J. curcas est également utilisé comme vermifuge : les graines grillées, mélangées aux arachides et macérées dans l'eau et bues immédiatement luttent contre les vers intestinaux. Les racines sont utilisées pour traiter les maladies gastroentériques chez les enfants de moins de sept ans. La décoction des racines soigne les vers intestinaux. L'infusion des feuilles prise en boisson et le massage de l'anus par les feuilles soignent les mycoses digestives chez les enfants. L'infusion des feuilles et de la tige traite également la jaunisse. Tandis que l'huile obtenue à partir des graines traite la teigne. Cette huile aurait des propriétés antifongiques. Jatropha curcas est utilisée aussi contre les infections sexuellement transmissibles. C'est ainsi que les graines écrasées et bues soit avec la bouillie ou à l'eau chaude traitent la gonococcie. Le gui (Tapinanthus sp.), un parasite de la plante est utilisé pour le traitement du mal gastrique. Il peut être simplement mâché, écrasé et pris dans la bouillie ou mélangé à l'eau. Le gui est très apprécié pour ses pouvoirs mystiques, car déposé sous le toit, il protège la famille contre toute éventuelle attaque par les sorciers.

Usages agroforestiers intégrant la plante Jatropha curcas $L$.

Jatropha curcas est intégré dans le système de production agropastorale et contribue à la protection des sols contre l'érosion, la protection des cultures, la délimitation des terrains et champs et joue le rôle de base foncière.

\section{Haies vives, clôtures végétales}

Jatropha curcas est utilisé comme clôture végétale des champs (Fig. 2) tels que le maïs (Zea mays), le mil (Sorghum sp.), l'arachide (arachis hypogea), le manioc (Manihot esculentus) ... pour empêcher les animaux en divagation de pénétrer dans ces champs. II sert par ailleurs de brise vent pour ces cultures : entoure les maisons et sert ainsi de clôture, protège les maisons contre les vents et les poussières. D'autres l'utilise également pour l'ombrage. Il est aussi utilisé pour clôturer les jardins de case. Les essences rencontrées généralement dans ces jardins sont : le manguier (Mangifera indica), l'anacardier (Anacardium occidentale), le papayer (Carica papaya), le citronier (Citrus sp.), le goyavier (Psidium goyava) et le bananier (Musa sp.). 
Tableau 2. Principaux groupes de maladies traitées par Jatropha curcas et les parties utilisées en médecine locale

Table 2. Main groups of diseases treated with Jatropha curcas and the parts used in local medicine

\begin{tabular}{|c|c|c|c|c|}
\hline Ethnies & Pathologies & \begin{tabular}{|l|} 
Proportion \\
$(\%)$
\end{tabular} & Organes utilisés & $\begin{array}{l}\text { Technique de } \\
\text { préparation }\end{array}$ \\
\hline \multirow{6}{*}{ Guidar } & Gonococcie & 12 & Fruits/Racines & $\begin{array}{l}\text { Poudre mélangée avec la } \\
\text { bouillie }\end{array}$ \\
\hline & Plaies/Brûlures & 46 & Tige/Feuilles/Racines & Extrait de sève \\
\hline & \multirow{2}{*}{$\begin{array}{l}\text { Parasitoses } \\
\text { intestinales/Dermatoses }\end{array}$} & 22 & Racines/Fruits & Décoction \\
\hline & & 10 & Feuilles/Graines & Infusion/macération \\
\hline & Trouble cardiaque & 04 & Gui & Macération \\
\hline & Maladies mystiques & 22 & Gui & $\begin{array}{l}\text { Macération/Dépôt du gui } \\
\text { sous le toit }\end{array}$ \\
\hline \multirow{4}{*}{ Daba } & Gonococcie & 12 & Fruits & Décoction \\
\hline & Plaies/Brûlures & 62 & Feuilles/tige & Extrait de sève \\
\hline & \begin{tabular}{|l|} 
Parasitoses \\
intestinales/Dermatoses
\end{tabular} & 38 & Feuilles & Infusion \\
\hline & Maladies mystiques & 12 & Gui & Dépôt du gui sous le toit \\
\hline \multirow{7}{*}{ Peuls } & \multirow{3}{*}{$\begin{array}{l}\text { Parasitoses } \\
\text { intestinales/Dermatoses }\end{array}$} & 32 & Feuilles & Macération \\
\hline & & 24 & Racines & Décoction \\
\hline & & 24 & Fruits & Onction d'huile \\
\hline & \begin{tabular}{|l|} 
Troubles cardiaques \\
\end{tabular} & 8 & Gui & Macération \\
\hline & Gastrite & 8 & Gui & Macération \\
\hline & Jaunisse & 18 & Feuilles/tiges & Infusion \\
\hline & Plaies/Brûlures & 26 & Tige & Extrait de sève \\
\hline \multirow[t]{2}{*}{ Guiziga } & $\begin{array}{l}\text { Parasitoses } \\
\text { intestinales/Dermatoses }\end{array}$ & 24 & Feuilles & Macération \\
\hline & Troubles cardiaques & 26 & Gui & Décoction \\
\hline
\end{tabular}

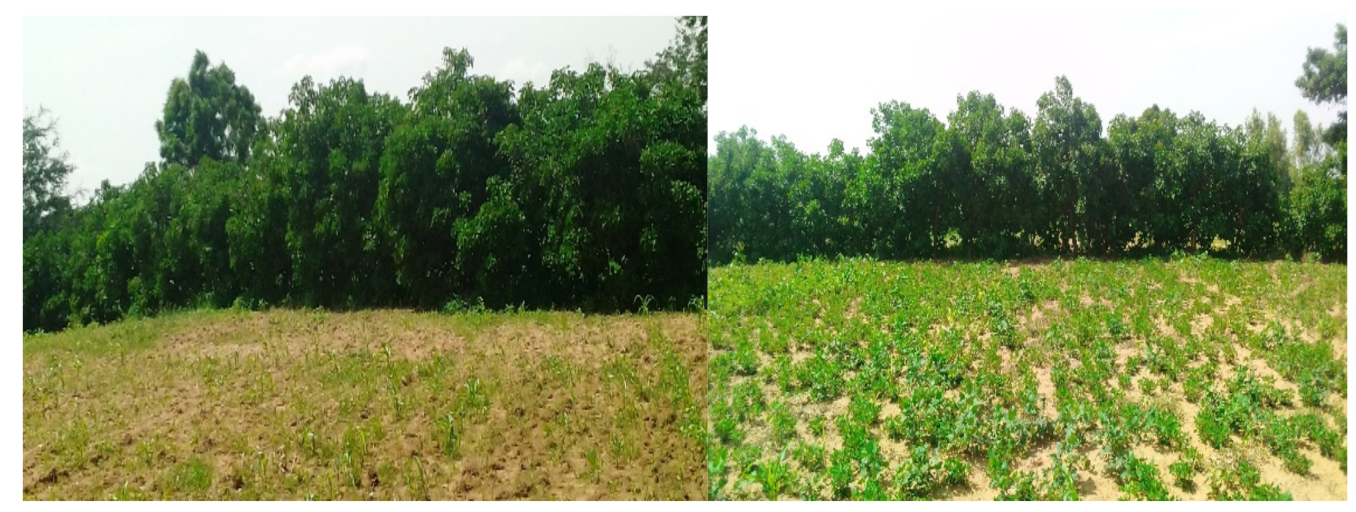

A

$\mathrm{B}$

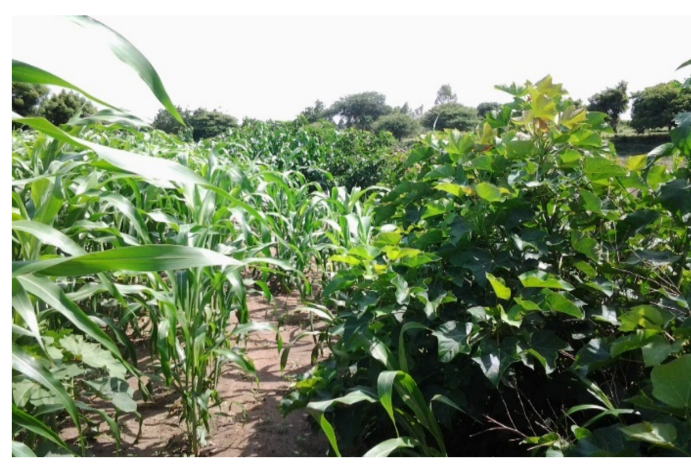

$\mathrm{C}$

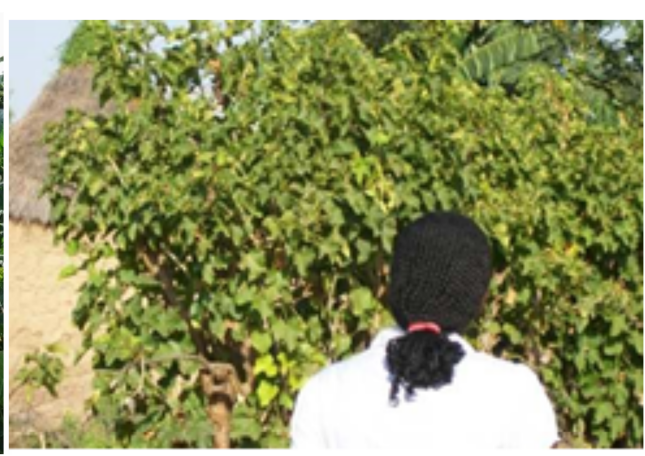

$\mathrm{D}$

Figure 2. Jatropha curcas utilisé comme clôture végétale : champs de maïs $(A)$, arachide $(B)$, mil $(C)$ et jardin de case (D)

Figure 2. Jatropha curcas used as plant fence: corn fields (A), peanuts (B), millet (C) and home garden (D) 


\section{Base foncière}

Pour les paysans, la plantation des arbres est un signe d'évolution, un indicateur de fixation, mais aussi un désir de laisser des traces de son passage. Le pourghère est souvent utilisé comme plantation des lisières et bornage. II borne quelquefois les pistes de sortie pour le bétail servant ainsi de corridor, empêchant ainsi les animaux d'entrer dans les champs. Certains paysans envisagent son utilisation pour clôturer les lieux de parcage de leurs bétails (bœufs, chèvres, moutons...). Toutes les ethnies enquêtées utilisent la plante pour marquer la limite des champs entre les voisins et marquent ainsi leur propriété servant de base foncière; ce qui permet d'éviter les conflits terriens.

Lutte contre l'érosion et conservation des sols Jatropha curcas est utilisé dans cette région pour la lutte antiérosive, mais aussi comme tuteur de certaines lianes et pour la protection contre les feux. Les haies vives de $J$. curcas luttent également contre l'érosion éolienne et hydrique. Les racines de la plante se développent à la surface du sol fixant ce dernier comme des digues ou des mottes de terre. Ces dernières freinent la dégradation du sol causée généralement par les eaux de ruissellement durant les fortes averses. Ce mécanisme permet ainsi aux paysans d'augmenter leur rendement agricole par la rétention des éléments nutritifs du sol.

\section{Caracteristiques botraniques de Jatropha curcas}

L.

Phénologie et fructification de Jatropha Curcas $L$.

Jatropha curcas n'est pas exigeant par rapport à la qualité du sol, car elle pousse sur tout type de sol, même sur les terres dégradées. La première floraison se déroule en début de saison pluvieuse, généralement en même temps que la feuillaison. La deuxième floraison intervient un à deux mois plus tard. II n'est pas rare de trouver chez un même arbuste des fruits à maturité, des fruits non mûrs et des inflorescences (Fig. 3). La défoliation se déroule en saison sèche, elle commence en novembre. Toutefois toutes les plantes ne perdent pas leurs feuilles simultanément. Ainsi dans certaines haies vives, il peut y avoir des plantes sans feuilles et des plantes avec feuilles.
(A)

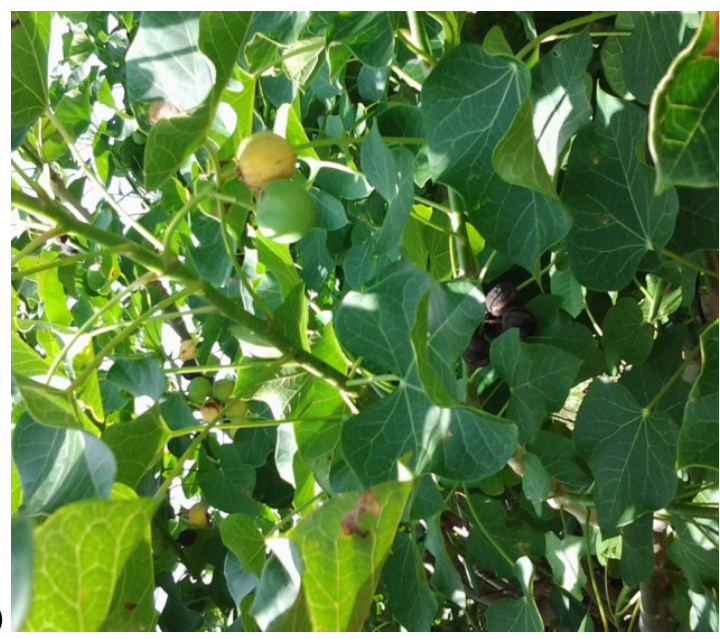

(B)

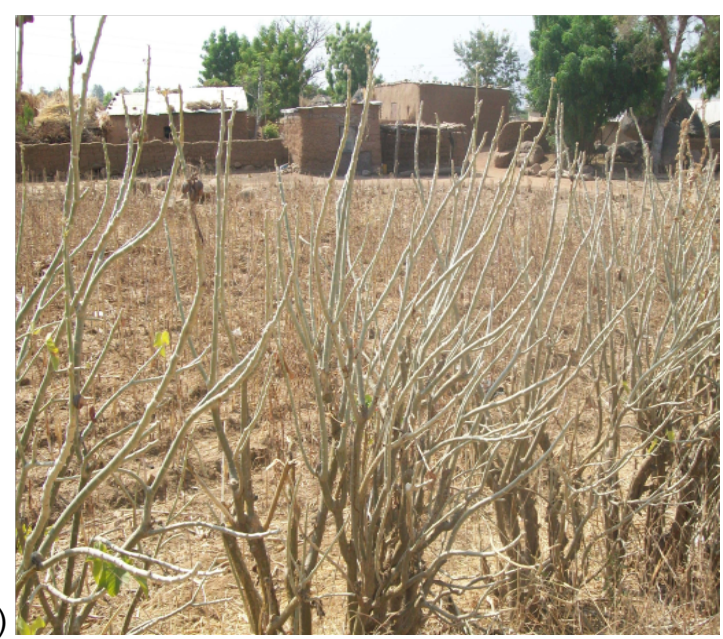

Figure 3. Spécimens de Jatropha curcas montrant des fruits immatures, matures et secs déhiscents (A) et une haie défoliée $(B)$

Figure 3. Specimens of Jatropha curcas showing immature, mature and dry dehiscent fruits $(A)$ and a defoliated hedge $(B)$

Les plants de Jatropha curcas, fructifient entre 1 et 3 ans. La majorité des personnes interviewées $(68,5 \%)$ affirme que la plante fructifie un an après plantation par multiplication végétative. Par ailleurs $67,75 \%$ des ménages interrogés déclarent que Jatropha curcas fructifie une seule fois par ans contre $32,25 \%$ pour ceux qui pensent plutôt que cette plante fructifie deux fois l'an (Tab. 3)

\section{Propagation et entretien de Jatropha curcas}

La propagation de Jatropha curcas se fait par bouture, semis et par transplantation (Tab. 4). Cependant la technique la plus utilisée est le bouturage (90,5\%). Les Guiziga n'emploient pas la transplantation. Les peuhls quant à eux utilisent uniquement le bouturage comme moyen de multiplication de J. curcas. 
Tableau 3. Âge et fréquence de fructification annuelle de Jatropha curcas Table 3. Age and annual fruiting frequency of Jatropha curcas

\begin{tabular}{|l|l|l|l|l|l|}
\hline & \multicolumn{3}{|c|}{ Âge de fructification (\%) } & \multicolumn{2}{c|}{ Nombre de fructification annuel (\%) } \\
\hline Ethnies & 1an & $\mathbf{2}$ ans & $\mathbf{3}$ ans & $\mathbf{1}$ fois & 2 fois \\
\hline Guidar & 62 & 24 & 14 & 62 & 38 \\
\hline Daba & 74 & 12 & 14 & 88 & 12 \\
\hline Peuhls & 54 & 36 & 10 & 54 & 46 \\
\hline Guiziga & 84 & 16 & 0 & 67 & 33 \\
\hline Moyenne & 68,5 & 22 & 9,5 & 67,75 & 32,25 \\
\hline
\end{tabular}

Tableau 4. Techniques de propagation de Jatropha curcas Table 4. Propagation techniques of Jatropha curcas

\begin{tabular}{|l|c|c|c|}
\hline & \multicolumn{3}{|c|}{ Modes de propagation (\%) } \\
\hline Ethnies & Semis & Transplantation & Bouturage \\
\hline Guidar & 4 & 6 & 90 \\
\hline Daba & 2 & 10 & 88 \\
\hline Peuhls & 0 & 0 & 100 \\
\hline Guiziga & 16 & 0 & 84 \\
\hline Moyenne & 5,5 & 4 & 90,5 \\
\hline
\end{tabular}

Caracterisation varietale de Jatropha curcas $L$.

La disposition spatiale de l'espèce Jatropha curcas montre un regroupement des plants autour des variables comme le diamètre à hauteur de poitrine $(\mathrm{DBH})$, la hauteur des arbustes (HAU), la longueur du pétiole (LPE) et la surface foliaire (SFO).
L'analyse des variances montre qu'il existe une corrélation significative entre la surface foliaire et la longueur du pétiole $(r=0,97 ; P<0,05)$ d'une part, puis entre le diamètre à hauteur de poitrine et la hauteur de l'arbuste $(r=0,87 ; P<0,05)$. La disposition de ces plants se fait suivant les axes $F_{1} \times F_{2}$ (Fig. 4).

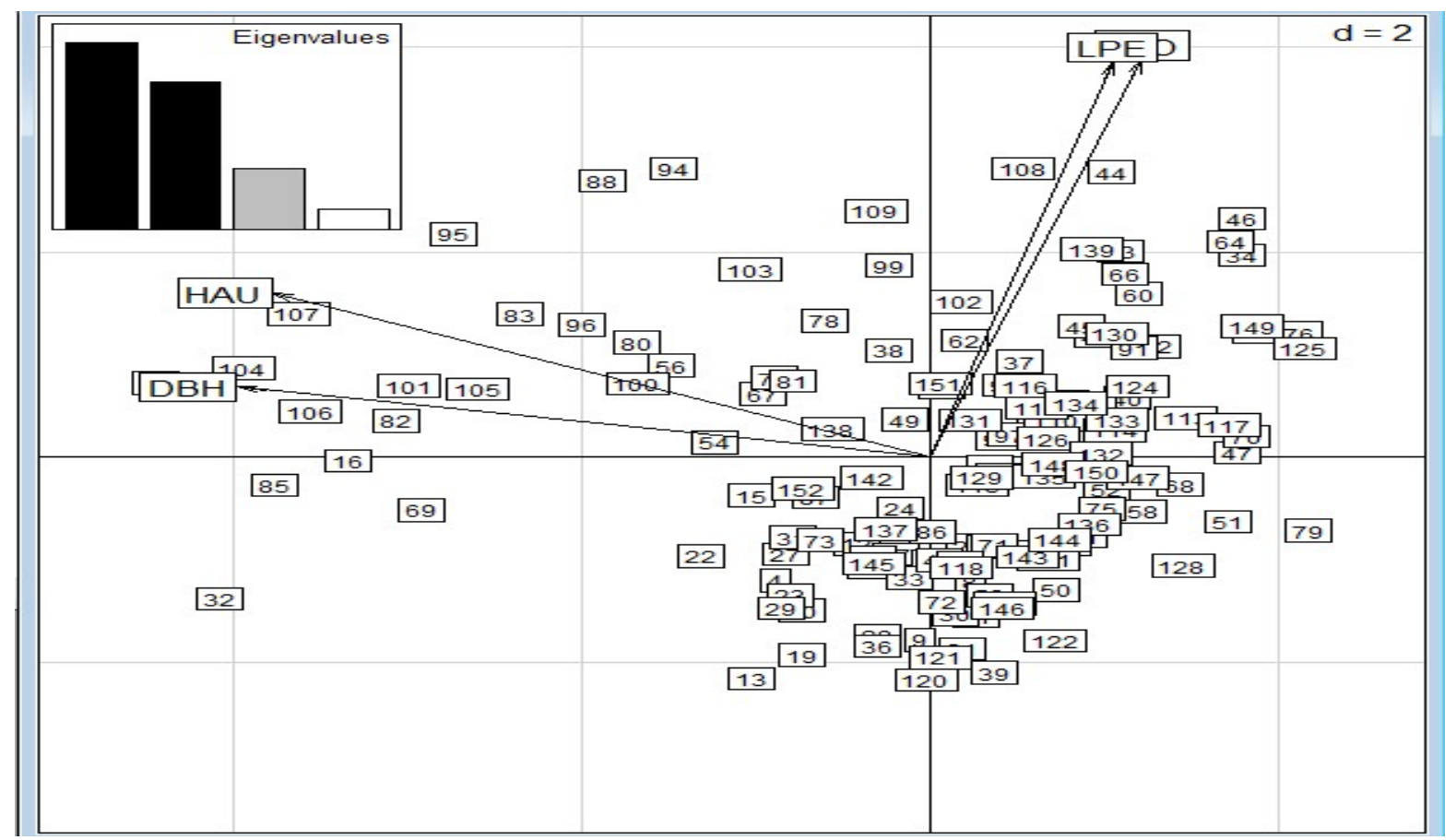

Figure 4. Analyse en composante principale des différentes variétés de Jatropha curcas Figure 4. Principal component analysis of the different varieties of Jatropha curcas 
L'analyse plus fine à la classification hiérarchisée révèle l'existence de quatre variétés lorsque l'indice d'homogénéité est pris à 500 (Fig. 5). À l'intérieur de chaque variété, il existe de sous-groupes ou sous variétés. Les caractères d'association sont les variables présentées ci-dessus. De gauche à droite de la figure 5 , on a : la variété 1 la moins représentée $(12,14 \%)$, la variété $2(34,28 \%)$, la variété 3 $(17,14 \%)$ et enfin la quatrième variété $(36,42 \%)$ la plus représentée à l'intérieur de la quelle existe divers sous-groupes.

La variété 1 (Tab. 5) présente la plus grande surface foliaire $\left(191,19 \mathrm{~cm}^{2}\right)$, la plus grande longueur de pétiole $(24,20 \mathrm{~cm})$ et la plus grande hauteur $(3,38 \mathrm{~m})$. Par contre la variété 4 possède la plus petite surface foliaire $\left(98,84 \mathrm{~cm}^{2}\right)$, la plus petite longueur du pétiole $(19,55 \mathrm{~cm})$ avec un plus grand diamètre à hauteur de poitrine $(8,34 \mathrm{~cm})$.

La variété 1 ne se rencontre pas dans la localité de Guider, la variété la plus rencontrée dans cette localité est la variété $4(92,30 \%)$. Par contre les trois autres variétés sont représentées dans les autres localités : Guider, Sorawel et Badjengo (Tab. 6).

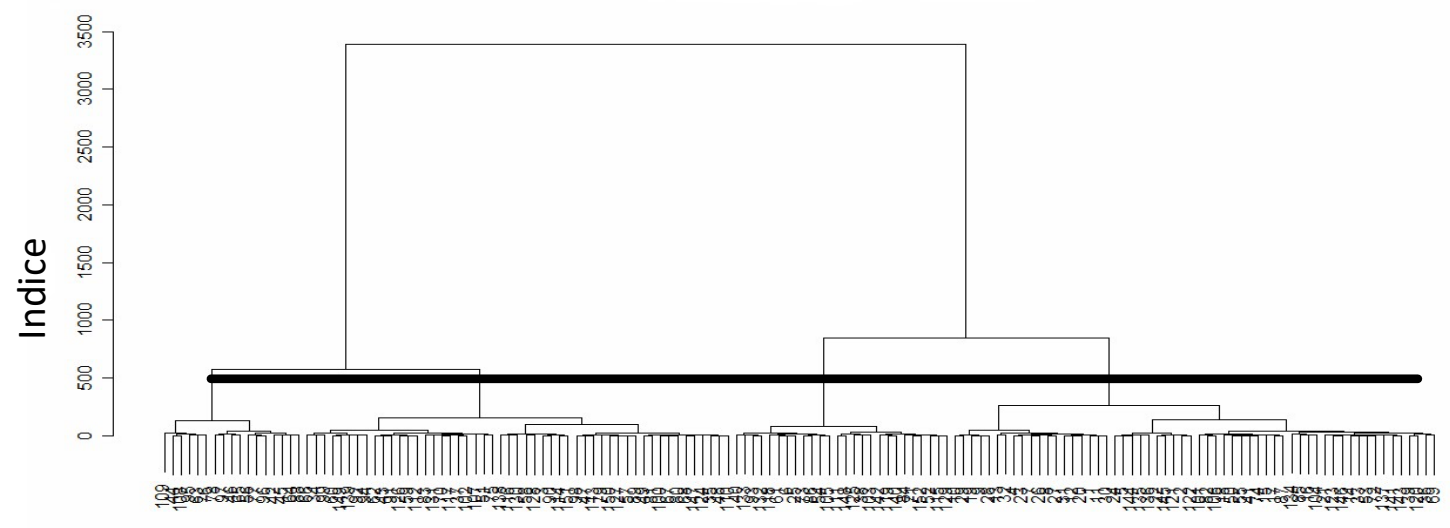

Figure 5. Dendrogramme montrant 4 types de variétés de Jatropha curcas Figure 5. Dendrogram showing 4 types of varieties of Jatropha curcas

Tableau 5. Paramètres des différentes variétés de Jatropha curcas Table 5. Parameters of the different varieties of Jatropha curcas

\begin{tabular}{|c|c|c|c|c|}
\hline & HAU $(\mathbf{m})$ & $\mathbf{S F O}\left(\mathbf{C m}^{\mathbf{2}}\right)$ & DBH $(\mathbf{m})$ & LPE $(\mathbf{c m})$ \\
\hline Variété 1 & 3,38 & 191,19 & 8,05 & 24,2 \\
\hline Variété 2 & 3,11 & 155,93 & 7,62 & 21,73 \\
\hline Variété 3 & 3,12 & 124,77 & 7,86 & 20,47 \\
\hline Variété 4 & 3,22 & 98,8 & 8,34 & 19,55 \\
\hline Moyenne & 3,20 & 142,67 & 7,96 & 21,48 \\
\hline
\end{tabular}

Tableau 6. Répartition (\%) des différentes variétés dans trois sites Table 6. Distribution (\%) of the different varieties in three sites

\begin{tabular}{|c|c|c|c|c|}
\hline Localités & Variété 1 & Variété 2 & Variété 3 & Variété 4 \\
\hline Guider & 0 & 3,84 & 3,84 & 92,3 \\
\hline Sorawel & 21,62 & 40,54 & 14,86 & 22,97 \\
\hline Badjengo & 2,5 & 42,5 & 30 & 25 \\
\hline Moyenne & 8,04 & 28,96 & 16,23 & 46,75 \\
\hline
\end{tabular}


La variété 1 est spécifique à Sorawel $(21,62 \%)$; elle possède la plus grande hauteur $(3,38 \mathrm{~m})$ et la plus grande longueur de pétiole $(24,2 \mathrm{~cm})$. La variété 2 est plus représentée à Badjengo $(42,5 \%)$ et à Sorawel $(40,54 \%)$. Cette variété possède la plus petite hauteur $(3,11 \mathrm{~m})$ et le plus petit diamètre à hauteur de poitrine $(7,62 \mathrm{~cm})$. La variété 3 quant à elle est plus représentée à Babjengo (30\%) et Sorawel $(14,86 \%)$. En suite la variété 4 la plus représentée à Guider (92,3\%), et caractérisée par la plus petite surface foliaire $\left(98,8 \mathrm{~cm}^{2}\right)$, la plus petite longueur de pétiole $(19,55 \mathrm{~cm})$ avec le plus grand diamètre à hauteur de poitrine $(8,34 \mathrm{~cm})$.

\section{Discussion}

L'origine du pourghère (Jatropha curcas) reste très peu connue dans la région du Nord-Cameroun, la provenance variant d'un groupe ethnique à l'autre. Quelques-uns l'ont cependant hérité de leurs parents. C'est le cas des Guidar (12\%) et des Daba $(30 \%)$ ou chez les éleveurs qui marquent leur trace par les arbres (Kossoumna 2008). Ceci s'expliquerait par le fait que ces peuples sont les autochtones de la localité ; $26,5 \%$ des ménages à l'exception des peuhls pensent qu'elle est d'origine sauvage et l'ont trouvé en brousse laissée par les éleveurs. En effet, le pourghère est une espèce pantropicale originaire d'Amérique Central. Des Caraïbes, il a été transporté dans d'autres pays d'Afrique et d'Asie par les pêcheurs portugais en passant par la Guinée Bissau (Jongschaap et al. 2007).

Presque tous les organes de la plante se prêtent à l'utilisation médicale (les feuilles, la tige, les racines, les fruits et le gui) avec différents modes de préparation (la décoction, l'infusion, la macération, l'extrait de sève et l'onction). Des multiples usages médicinaux de Jatropha curcas ont été relevés par plusieurs auteurs (Melanie \& Ruppel, 2000, Alfons 2007, Fézan et al. 2008). Jatropha curcas est utilisée dans le traitement de l'hypertension artérielle et le diabète en Côte d'Ivoire (Fezan et al. 2008). Lorsque le gui (Tapinanthus sp.) de Jatropha curcas est mis sous la toiture, il protège l'utilisateur contre plusieurs maux mystiques y compris l'empoisonnement. Ce pouvoir mystique a été également signalé chez les Frofro baka en Côte d'Ivoire et en Haïti chez les Godiés (Alfons 2007, Fézan et al. 2008, Melanie \& Ruppel 2000). Malgré l'importance des vertus médicinales du gui (Tapinanthus sp.) prélevé sur J. curcas, ce parasite peut occasionner la mort de la plante. Les stratégies d'attaque de ce parasite ont été rapportées par Mapongmetsem et al. (1997) dans les savanes soudano-guinéennes du Cameroun. Au Bénin J. curcas est utilisé dans le traitement de certaines maladies similaires de notre zone d'étude telles que : la gonococcie, le mal de ventre, la jaunisse et les plaies (Assogbadjo et al. 2009). Les graines ou I'huile de Jatropha curcas est un purgatif ou laxatif et sa racine est utilisée contre la lèpre, le latex pour arrêter le saignement et lutter contre les infections, les feuilles pour traiter le paludisme. Cette plante semble contenir des principes actifs ayant une activité désinfectante, antifongique et antiparasitaire, susceptible d'être utilisée contre la Malaria comme les baies de raisin d'Amérique. L'extrait de cette plante serait un excellent molluscide, actif contre l'escargot hôte de Schistosoma mansoni et Schistosoma haematobium, vecteurs de la bilharziose (Jongschaap et al. 2007). Les propriétés médicinales reconnues aux différents organes de Jatropha curcas font de cette plante une source potentielle pour la valorisation de la pharmacopée africaine et celle du Cameroun en particulier. Mais toutefois une étude judicieuse sur la toxicité de cette essence serait nécessaire pour l'élaboration future des médicaments améliorés à partir de cette plante.

L'intégration de cette essence végétale dans le système agroforestier a été rapporté à Madagascar, en Afrique centrale, dans les grands lacs et en Afrique de l'Ouest (Alfons 2007, König 1992, Molenaar \& Kessler, 2008). Jatropha curcas est utilisé à Madagascar comme haie de clôture des fermes, des champs ou du parc à bœuf, délimitation des propriétés mitogènes, haie de protection contre l'érosion par le vent, l'eau et les feux de brousse (Alfons 2007). Grâce au développement rapide de J. curcas, l'érosion est réduite. Ce qui permet de réduire les pertes en matière organique et en éléments nutritifs à un niveau tolérable (König 1992). Lors de fortes pluies, les parcelles agroforestières situées en plaine jouent un rôle pour les embâcles et ralentissent le courant (Alfons, 2007). L'intégration des arbres en général comme base foncière a été relevé par plusieurs auteurs (Mapongmetsem 2005, Kossoumna 2008) et en particulier de Jatropha curcas (Alfons 2007). Cette difficulté à déterminer le nombre de fructification en une année viendrait du fait que toutes les branches ne fleurissent pas de manière synchrone. Ce constat est conforme à celui fait par Henning (2000). Habituellement la propagation par bouturage est la plus utilisée car la régénération par bouturage est plus rapide que celle issue des semis. Ces résultats sont en accord avec ceux trouvés par Domergue et Piror (2008).

Jatropha curcas est répandue dans la région du Nord-Cameroun et plusieurs ménages intègrent cette plante dans leur système de production, malheureusement ses graines ne sont pas bien valorisées car elles sont rejetées dans la nature à part leur usage médicinal. Le développement de la filière biocarburant à partir des graines de Jatropha curcas pourra réduire les effets du changement 
climatique et la dépendance des pays en voie de développement vis-à-vis des énergies fossiles notamment les hydrocarbures qui sont des ressources énergétiques non renouvelables (Gbosségnon, 2007). Plusieurs auteurs ont relevé les potentialités de Jatropha curcas comme biocarburant (Jongschaap et al., 2007 ; Gbosségnon, 2007; Alfons, 2007). L'huile de Jatropha est utilisable sans raffinage comme carburant de moteurs diesels et comme huile lampant. Cette huile brûle nettement plus lentement que le pétrole et dégage moins de fumée, ce qui est un avantage pour l'éclairage de chambres fermées. Par ailleurs le développement de la filière biocarburant pourra créer des revenus aux populations locales (la vente des graines, la fabrication du savon).

Quatre différentes variétés de Jatropha curcas ont été dénombrées dans les localités de Guider, Sorawel et Badjengo en fonction des critères dendrométriques (le diamètre à la hauteur de poitrine, la hauteur de l'arbuste, la surface foliaire et la longueur du pétiole). Cette méthode dendrométrique plus particulièrement le phénogramme a été utilisé par Heller (1996) ; ce qui lui a permis de dénombrer 77 espèces néotropicales du genre Jatropha à partir de 32 caractères. Abdoulaye et Yacouba (2013) au Mali quant à eux ont utilisé la sélection massale pour obtenir deux écotypes de Jatropha curcas, variétés un peu mieux adaptées pour la production de fruits (écotype Konséguéla et l'écotype de Dogo).

\section{Conslusion}

L'origine de Jatropha curcas reste méconnue dans la région du Nord-Cameroun malgré les multiples usages reconnus à cette essence végétale. II possède plusieurs vertus médicinales. Ainsi, il traite plusieurs maladies : la gonococcie, les blessures, les parasitoses intestinales, le trouble cardiaque, et les maladies mystiques. Toutes les parties de la plante se prêtent à l'usage médicinal (la tige, les feuilles, les racines, gui). Les modes de préparation les plus utilisés sont l'infusion, la décoction, la macération, l'onction et l'extrait de sève. Jatropha curcas est de même intégré dans les systèmes agroforestiers et joue plusieurs rôles dans la production agropastorale : lutte antiérosive, haies vives, clôtures végétales et base foncière. II existe quatre variétés de Jatropha curcas dans la région avec des caractéristiques différentes. Des analyses moléculaires pourront permettre d'approfondir l'analyse taxonomique dans la perspective de promouvoir la variété la plus performante. La valorisation de cette plante serait très bénéfique pour la communauté locale et internationale (la pharmacopée traditionnelle, la création d'emplois par la vente des graines, la restauration des terres dégradées grâce à l'intégration de cette plante dans les systèmes agroforestiers et la lutte contre les changements climatiques).

\section{Déclarations}

Approbation éthique et consentement à participer: Toutes les données utilisées dans cet article n'ont jamais été publiées dans des revues ; les personnes interviewées étaient totalement consentantes et ont donné leur accord pour participer aux enquêtes.

Consentement à la publication: Sans objet, aucune donnée personnelle n'est incluse dans ce manuscrit.

Disponibilité des données et du matériel: Les données brutes sont disponibles auprès des auteurs. Conflit d'intérêts: les auteurs déclarent ne pas avoir d'intérêts concurrents.

Financement: Cette étude n'a pas reçu de financement.

Contributions des auteurs: Le travail de terrain, la collecte des données et la rédaction de ce travail ont été réalisés par $A B$. La supervision et l'analyse des données ont été faites par MPM. Tous les auteurs ont lu, corrigé et approuvé le manuscrit.

Remerciements: Les auteurs remercient particulièrement les paysans qui ont montré un intérêt particulier au Jatropha et pour avoir accepté de partager leurs connaissances. En outre, ils sont reconnaissants aux reviewers anonymes et à l'éditeur Dr. Rainer W. Bussmann pour leurs commentaires constructifs.

\section{Litérature citée}

Abdoulaye VS, Yacouba C. 2013. CIRAD. Synthèse bibliographique sur le Jatropha au Mali $20 \mathrm{p}$.

Alfons Ü., 2007. Jatropha à Madagascar- Rapport sur l'état actuel du secteur. Gesellschaft für Technische Zusammenarbeit (GTZ) Madagascar 32 p.

Assogbadjo AE, Amadji G, Kakaï RG, Mama A, Sinsin B, Damme PV. 2009. Evaluation écologique et ethnobotanique de Jatropha curcas L. au Bénin. International Journal of Biological and Chemical Sciences 3(5):1065-1077.

Ballerini D. 2006. Les biocarburants : perspectives, risques et opportunités. Edition Technip 190 p.

Berchmans HJ, Hirata S. 2008. Biodiesel production from crude Jatropha curcas L. seed oil with a high content of free fatty acids. Bioresource Technology 99:1716-1721.

Domergue M, Piror R. 2008. Jatropha curcas L. Rapport de synthèse bibliographique, Agrogénération $118 \mathrm{p}$. 
Fézan $\mathrm{H}$, Tra Bi, Guy M, Irié K, N'gaman, Clejesson $\mathrm{HB}$, Mohou. 2008. Études de quelques plantes thérapeutiques utilisées dans le traitement de l'hypertension artérielle et du diabète : deux maladies émergentes en Côte d'Ivoire. Sciences \& Nature 5(1):39- 48.

Gbosségnon C. 2007. Situation des biocarburants en Afrique de l'Ouest. Extrait du RIAED/Réseau international d'accès aux énergies durables $4 p$.

Heller J. 1996. Physic nut. Jatropha curcas L. in International Plant Genetic Resources Institute (IPGRI), Promoting the conservation and use of underutilized and neglected crops. Prom Underused Crops 1:1-66.

Henning R. 2000. Use of Jatropha curcas oil as raw material and fuel: an integrated approach to create income and supply energy for rural development. Experiences of the Jatropha Project in Mali, West Africa. Presentation at the International Meeting "Renewable 15 Energy. A Vehicle for Local Development-II". Folkecenter for Renewable Energy, Denmark $5 \mathrm{p}$.

Humbel FX. 1971. Carte pédologique de Ngaoundéré. Centre de Yaoundé, Cameroun. ORSTOM, Yaoundé, Cameroun. Note explicative $118 \mathrm{p}$.

Jongschaap REE, Corré WJ, Bindrahan PS, Bandenburg WA. 2007. Calims and facts on Jatropha curcas $66 \mathrm{p}$.

König D. 1992. L'agriculture écologique agroforestière, une stratégie intégrée de conservation des sols au Rwanda. Bull. Réseau Erosion, IRDMontpellier 12:130-139.

Kossoumna LN. 2008. De la mobilité à la sédentarisation: Gestion des ressources naturelles et des territoires par les éleveurs Mbororo au Nord du Cameroun Thèse de Doctorat en géographie de I'Université Paul Valéry, Montpellier III 284 p.

Melanie Rug, Andreas Ruppel. 2000. Toxic activities of the plant Jatropha curcas against intermediate snail hosts and larvae of schistosomes. Tropical Medicine and International Health 6:423-430.

Mapongmetsem PM, Motalindja MH, Nyomo. 1997. Eyes enemy: parasites of fruits trees in the sudanoguinean savannah. AF. Todays $19 \mathrm{p}$.

Mapongmetsem PM. 2005. Phénologie et apport au sol des substances biogènes par la litière de quelques fruitiers sauvages des savanes soudanoguinéennes Thèse de Doctorat d'Etat, Université de Yaoundé I $267 \mathrm{p}$.

Mapongmetsem PM, Tchiegang-Megueni C, Nkongmeneck BA, Kapseu C, Kayem GJ. 1997. Agrofroestry potentials of the indigenous tree species in northern Cameroon. Journal of Biochemistry Science 7 (1):24-29.
Molenaar JW, Kessler JJ. 2008. CATALIST: Catalyser I'Intensification Agricole Accélérée pour la Stabilité Sociale et Environnementale dans la Région des Grands Lacs de l'Afrique Centrale, Manuel de formation sur l'Agroforesterie dans le cadre de l'intensification agricole, Kigali $43 p$.

Noumi E.\& Anguessin B. 2010. Insecticide and ethnomedecine of HIVIAIDS at Tokombere (Far North Cameroon). Indian Journal of Traditional Knowledge 9(3):730-735.

Sokpon N, Lejoly J. 1996. Les plantes à fruits comestibles d'une forêt semi-caducifoliée : Pobè au Sud-est du Bénin. L'alimentation en forêt tropicale, Interactions bioculturelles et perspectives de développement. Les ressources alimentaires : production et consommation. Editions UNESCO MAB, Pp. 315-324.

Torquebiau E. 2007. L'agroforesterie: des arbres et des champs. L'Harmattan. Paris. 